\title{
Estudio de la acuatermólisis catalítica en procesos de upgrading de crudos pesados como método complementario en el recobro térmico de hidrocarburos
}

\author{
Jorge Anderson Arboleda Lamus *; Ángel Mauricio Castillo Cubillos; Samuel Fernando Muñoz Navarro \\ Grupo de Investigación Recobro Mejorado. Universidad Industrial de Santander (UIS), Bucaramanga, Colombia. \\ *E-mail: zone.ander@gmail.com
}

\begin{abstract}
Resumen
La densidad y viscosidad es la propiedad que diferencia el petróleo liviano del pesado y extrapesado; el petróleo pesado y extrapesado contiene más constituyentes indefinidos químicamente (resinas y asfaltenos) que uno que tenga más constituyentes puros (saturados y aromáticos). En la inyección de vapor se reduce la viscosidad en un 97\%; además, la implementación de vapor de agua también cambia la composición debido a reacciones químicas entre el vapor de agua, los compuestos orgánicos del petróleo, los minerales y catalizadores, logrando un cambio de la estructura molecular del petróleo, este proceso es definido como acuatermólisis catalítica. Las reacciones de acuatermólisis catalítica son: pirolisis, hidrolisis, reacción de gas al agua (W.G.S. por sus siglas en inglés), hidrodesulfuración (HDS), hidrodesnitrogenación (HDN) e hidrodesoxigenación (HDO). El uso de catalizadores metálicos y donadores de hidrógeno presentan un efecto sinérgico, además que los compuestos que reaccionan con el vapor de agua son las resinas y los asfaltenos. A escala de laboratorio se logra reducir el $90 \%$. A escala de campo, se han realizado pilotos en la cuenca de Liaohe, China, donde se utilizó como complemento a la tecnología de inyección cíclica de vapor, mostrando reducciones en la viscosidad del $80 \%$.
\end{abstract}

Palabras clave: Acuatermólisis, Catalizadores, Vapor, Viscosidad, Saturados, Aromáticos, Resinas, Asfaltenos.

\section{Study of catalytic aquathermolysis in processes of upgrading of heavy petroleums as a complementary method in the hydrocarbons thermal recovery}

\begin{abstract}
Density and viscosity are the properties which differentiates light oil of the heavy and extra-heavy oil; heavy-extra-heavy oil contains more indefinite chemical elements such as resins and asphaltenes whose have more pures components (saturated and aromatics). In the steam injection, the viscosity is reduced at $97 \%$, in addition, the implementation of steam also changes the composition of the mixure due to chemical reactions between the water steam, organics petroleum compounds, minerals and catalysts, reaching a switch in the molecular structure of petroleum; this process is defined as catalytic aquatermolysis. The reactions of catalytic aquatermolysis are: pyrolysis, hydrolysis, Water Gas Shift, hydrodesulphurization (HDS), hydrodesnitrogenación (HDN) and hidrodeoxygenation (HDO). The use of metal catalysts and hydrogen donors have a synergistic effect, in addition, the compounds that react with water steam are resins and asphaltenes. On a macro scale, it is reduced by $90 \%$. At the field scale, pilots were implemented in the Liaohe basin, China, where it was used as a complement to the cyclic steam injection technology, showing reductions in viscosity of $80 \%$ and relevant changes in the composition of the oil.
\end{abstract}

Keywords: Aquathermolysis, Catalytic, Steam, Viscosity, Saturated, Aromatics, Asphaltenes.

Cita: Arboleda, J. A., Castillo, A. M. y Muñoz, S. F. (2018). Estudio de la acuatermólisis catalítica en procesos de upgrading de crudos pesados como método complementario en el recobro térmico de hidrocarburos. Revista Fuentes: El reventón energético, vol 16(1), 57-69. 


\section{Introducción}

La disminución de las reservas de petróleo convencional a nivel mundial incentiva a las compañías petroleras a invertir en los yacimientos de crudos pesados y extrapesados. Dos factores son importantes para el auge de la explotación de este tipo petróleo: actualmente existen varias técnicas que permiten su recuperación y la disponibilidad de estos recursos. El total de recursos de petróleo mundial se aproxima de 9 a 13 trillones de barriles, de los cuales el 70\% corresponde a petróleo no convencional: $30 \%$ arenas petrolíferas y bitumen $\left(<8{ }^{\circ} \mathrm{API}\right), 25 \%$ de petróleo extrapesado $\left(<10{ }^{\circ} \mathrm{API}\right)$ y petróleo pesado ( $<20$ API) (Alboudwarej, Felix \& Taylor, 2006).

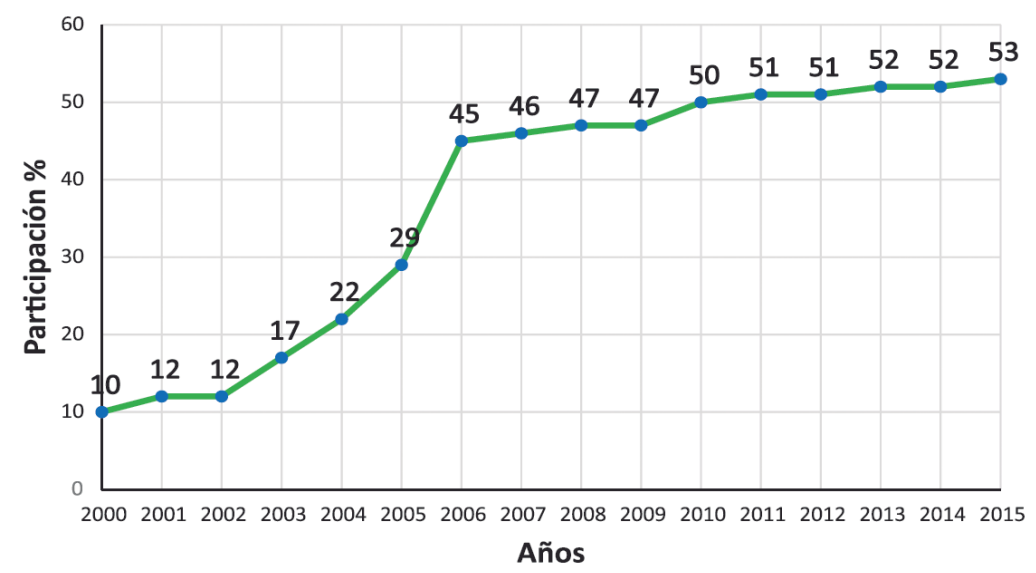

Figura 1. Participación de petróleo pesado y extrapesado en producción de Colombia. Fuente: Molina (2015).

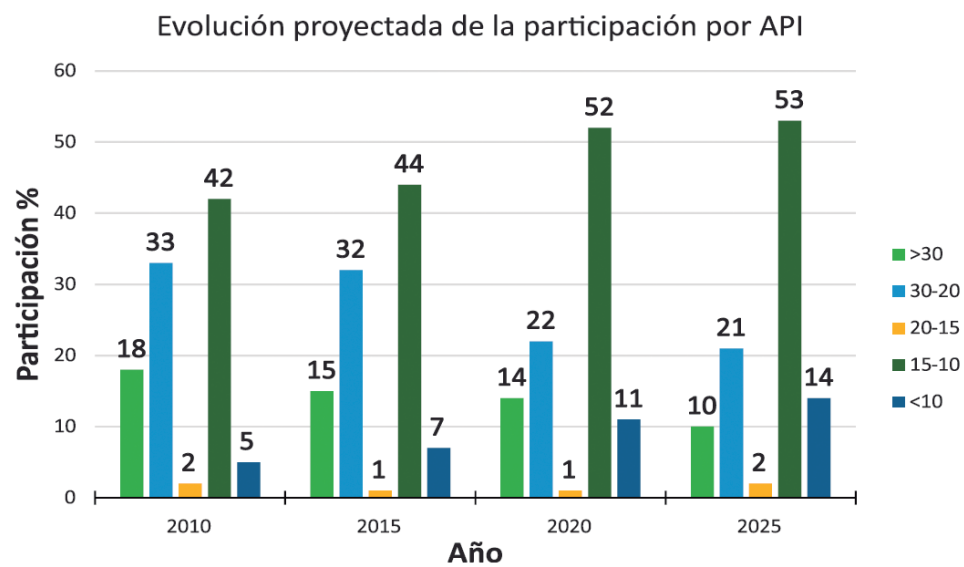

Figura 2. Proyección de la participación por API. Fuente: Molina (2015).

En el panorama nacional, la fig. 1, representa la participación de petróleo pesado y extrapesado en la producción de Colombia. En 2015, la producción colombiana fue de 367 millones de barriles; y, de acuerdo con Ecopetrol, la contribución del petróleo pesado y extrapesado a la producción del 2015 fue de 194 millones de barriles (53\%) (Molina, 2015). La fig. 2, muestra la proyección de la participación de petróleo por ${ }^{\circ} \mathrm{API}$ en la producción petrolera en Colombia. Como se aprecia, al 2025; la participación de este será del $69 \%$, esto ratifica que el futuro de la industria petrolera en Colombia y en el mundo, se moverá en el desarrollo de nuevas técnicas y tecnologías para la extracción de este tipo de hidrocarburos.
La recuperación primaria y secundaria en los yacimientos de petróleo extrapesado comúnmente suele ser muy baja, es decir, la cantidad de petróleo original in-situ recuperado por flujo natural, y por los mecanismos de levantamiento artificial o inyección de agua es poca (Cavanzo, Navarro, Ramírez, Niz \& Rodríguez, 2016). Es usual que crudos pesados que se encuentren a una temperatura de $43{ }^{\circ} \mathrm{C}$ tengan viscosidades superiores a $2000 \mathrm{cP}$ y que, al aumentar su temperatura a $104{ }^{\circ} \mathrm{C}$, este haya reducido su viscosidad a solo $60 \mathrm{cP}$ (Ahmed, 2012). Los métodos de recobro térmico (inyección de vapor y combustión in-situ) presentan un gran potencial en el mejoramiento de la recuperación de petróleo pesado y extrapesado, ya que aumentan la temperatura 
del yacimiento al inyectar un fluido caliente que transfiere su energía térmica al petróleo y por ende reduce su viscosidad (Ahmed, 2012).

En el presente artículo, se abordará la acuatermólisis catalítica como método complementario a la técnica de inyección de vapor y se presenta el potencial que podría alcanzar en el auge del petróleo pesado y extrapesado. Se plantearán los conceptos básicos para comprender la Acuatermolisis Catalítica y se finaliza con la revisión de unos artículos donde se muestra la eficiencia técnica y tecnológica de la misma.

\section{Marco teórico}

El petróleo es una mezcla de constituyentes puros (hidrocarburos, orgánicos de heteroátomos y organometálicos) y compuestos indefinidos químicamente (resinas y asfaltenos) (Wauquier, 2004); la diferencia principal entre el petróleo pesado y extra pesado del petróleo liviano es la densidad y viscosidad; esa diferencia es producida por la composición: la relación de constituyentes puros y compuestos indefinidos químicamente, entre más constituyentes indefinidos químicamente tenga el petróleo mayor será su densidad. Esto también genera un fuerte incremento en la viscosidad del crudo.

En los últimos años la industria petrolera se ha enfocado en el desarrollo de nuevas técnicas que permitan la extracción de petróleo pesado y extrapesado. La inyección de vapor es una técnica térmica de recobro mejorado empleada en yacimientos de petróleo pesado y extrapesado (Alarcón y Cavanzo, 2013). El mecanismo principal de la inyección de vapor es la reducción de la viscosidad del crudo hasta de 33 veces debido a la transferencia de calor (Ahmed, 2012), esta reducción de la viscosidad afecta positivamente la relación de movilidad (movilidad del fluido desplazante sobre el desplazado) y como consecuencia se disminuye la saturación de aceite en el yacimiento (Mercado, 2008). Existen otros mecanismos que están presentes en la inyección de vapor (Ahmed, 2012), estos son:
- Expansión térmica e hinchamiento del aceite

- Destilación al vapor

- Empuje por gas en solución

- Desplazamiento miscible.

Además de los mecanismos expuestos, los compuestos orgánicos pueden sufrir cambios en su estructura molecular al inyectar vapor de agua al yacimiento. Roa (2014), y (Kapadia, Kallos \& Gates, 2014), clasifican las reacciones de vapor de agua y compuestos orgánicos en rangos de altas temperaturas $\left(>300^{\circ} \mathrm{C}\right)$ y bajas temperaturas $\left(<300^{\circ} \mathrm{C}\right)$ (Villamarín, Cañas \& Navarro, 2014).

A altas temperaturas se logran reacciones de "pirolisis" en la cual, la acción de calentar el crudo en ausencia de oxígeno hace que las moléculas más grandes principalmente resinas y asfaltenos se rompan y generen radicales de heteroátomos $(\mathrm{S}, \mathrm{N}$ y $\mathrm{O})$ y moléculas de menor tamaño (Murazan \& Galadima, 2015); estas reacciones ocurren en presencia o no de agua (Roa, 2012). Se ha demostrado que, con el tiempo, los radicales de heteroátomos pueden sufrir reacciones de polimerización para producir moléculas más grandes, de esta manera, aumenta la viscosidad y densidad del crudo; este proceso es conocido como reversibilidad de la viscosidad y es adverso (Murazan \& Galadima, 2015).

"Acuatermólisis" es el proceso de reacciones bajas temperaturas, el cual se define como la interacción química del vapor de agua y compuestos orgánicos del petróleo crudo en presencia de calor y ausencia de oxígeno, en el que se logra un cambio en la estructura molecular de los compuestos orgánicos (Castillo, 2017). Las reacciones de acuatermólisis son: pirolisis, hidrólisis, reacción del gas al agua (W.G.S.), hidrodesulfuración (HDS) (Murazan \& Galadima, 2015); estas dos últimas consisten en la reducción del contenido de heteroátomos de oxígeno y azufre, donde se produce dióxido de carbono $\mathrm{CO}_{2}$, monóxido de carbono $\mathrm{CO}$, ácido sulfhídrico $\mathrm{H}_{2} \mathrm{~S}$, hidrógeno $\mathrm{H}_{2}$ e hidrocarburos livianos (Murazan \& Galadima, 2015). Las reacciones se presentan en un intervalo de temperaturas entre $200^{\circ} \mathrm{C}$ y $300^{\circ} \mathrm{C}$ (Roa, 2012).

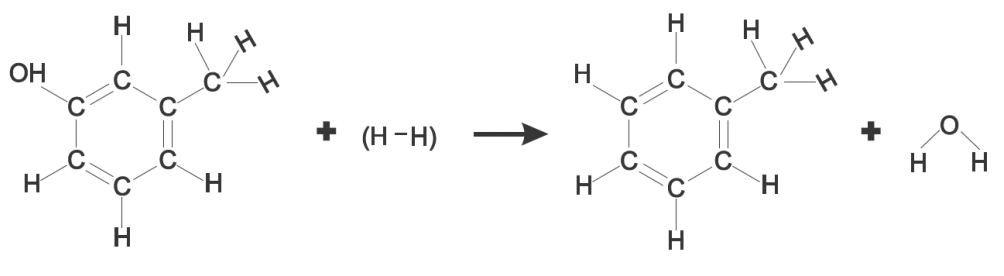

2-metilfenol Hidrógeno Tolueno Agua

Figura 3. Sub-proceso de hidrodesoxigenación por reacciones de acuatermólisis catalítica (Adicion de hidrógeno).

Fuente: Gonzales (2014). 
Adicionar catalizadores puede mejorar la reacción de pirolisis al disminuir la energía de activación para romper los enlaces carbono-carbono C-C, carbonoazufre C-S, carbono-nitrógeno C-N y carbono-oxígeno C-O (Cañas, Ramírez \& Navarro, 2014). Asimismo, en muchos casos se hace necesaria la implementación de agentes donadores de hidrógeno, cuyo fin es evitar la polimerización de los radicales libres producto de la pirolisis de resinas y asfaltenos, ya que, en ocasiones el agua no es suficiente para suplir la cantidad de hidrógeno necesario. La interacción de compuestos orgánicos y vapor de agua en presencia de catalizadores y minerales del yacimiento da como resultado el término "acuatermólisis catalítica" (Murazan \& Galadima, 2015). La acuatermólisis catalítica está compuesta por dos procesos: W.G.S. e hidrotratamiento, los cuales son llevados a cabo por las reacciones de: pirolisis, hidrólisis, W.G.S., (HDS), hidrodesulfuración (HDS), hidrodesnitrogenación (HDN) e hidrodesoxigenación (HDO), cuyos productos son dióxido de carbono $\mathrm{CO}_{2}$, monóxido de carbono $\mathrm{CO}$, ácido sulfhídrico $\mathrm{H}_{2} \mathrm{~S}$, hidrógeno $\mathrm{H}_{2}$ e hidrocarburos livianos, amoniaco NH3 y agua $\mathrm{H}_{2} \mathrm{O}$ (Murazan \& Galadima, 2015); las reacciones HDS, HDN e HDO hacen parte del proceso de hidrotratamiento (Garzón, Cañas \& Navarro, 2012).

a)

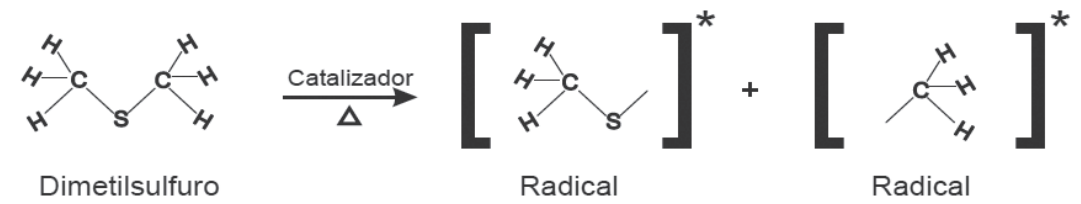

b)
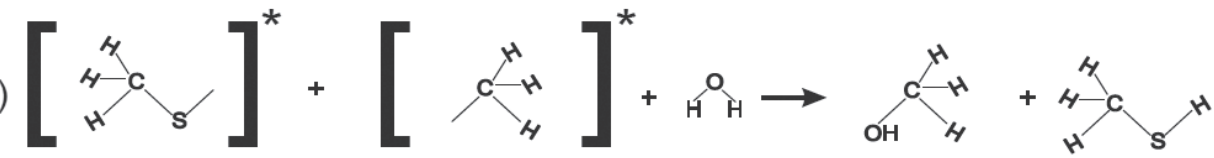

Radical

Radical

Agua

Metanol

Metanotiol

c)

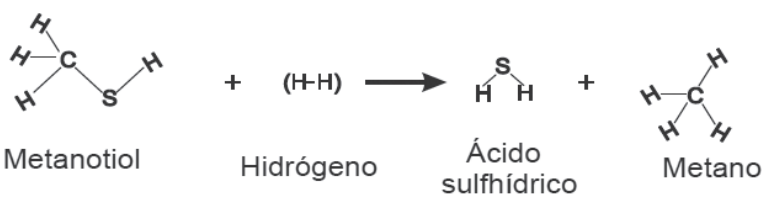

Figura 4. Sub-proceso de hidrodesulfuración por reacciones de acuatermólisis catalítica. a) pirolisis, b) hidrolisis e c) hidrodesulfuración. Fuente: Roa (2012).
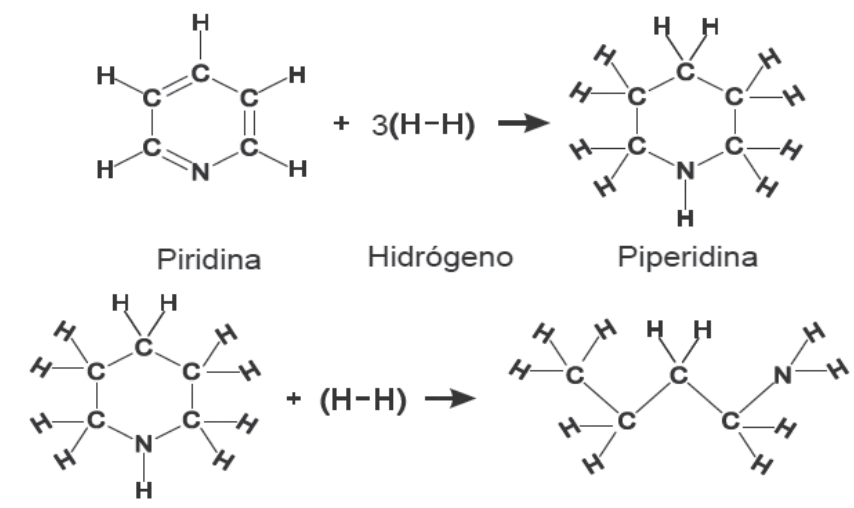

Piperidina Hidrógeno n-Pentilamina

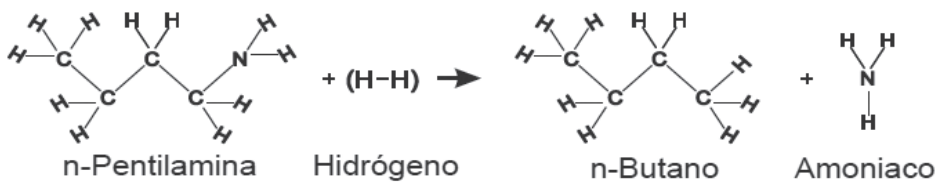

Figura 5. Sub-proceso de hidrodesnitrogenación por reacciones de acuatermólisis catalítica (adición de hidrógeno).

Fuente: Valdevinito et al. (2010). 
Las fig. 3, 4 y 5 representan los sub-procesos del hidrotratamiento, para cada heteroátomo presente en la molécula de asfalteno. A continuación, se enumeran las consecuencias de la implementación de acuatermólisis catalítica (Acevedo, 2010):

- Cambio estructural permanente del crudo tratado.

- Reducción del peso molecular promedio.

- Reducción de la viscosidad.

- Reducción del contenido de asfaltenos y resinas.

- Reducción del contenido de azufre, carbono, nitrógeno y metales.

- Incremento de hidrocarburos saturados y aromáticos.

- Aumento en la saturación de gases de ácido sulfhídrico, amoniaco y dióxido de carbono.
De la acuatermólisis el proceso de W.G.S. es el más importante, ya que en él se desoxigena las moléculas y se produce $\mathrm{CO}, \mathrm{H}_{2}$ y $\mathrm{CO}_{2}$ (Acevedo, 2010). El $\mathrm{H}_{2}$ y $\mathrm{CO}_{2}$ se produce a partir de la reacción de $\mathrm{CO}$ y $\mathrm{H}_{2} \mathrm{O}$. La ecuación 1 muestra la reacción reversible de $\mathrm{CO}$ y $\mathrm{H}_{2} \mathrm{O}$, mientras la fig. 6 representa el proceso de W.G.S. El consumo del $\mathrm{H}_{2}$ en otras etapas de la acuatermólisis (HDS, HDN e HDO), favorece la producción de más $\mathrm{CO}_{2}$ y de más $\mathrm{H}_{2}$ (Rojas, Cañas \& Navarro, 2012). Por otra parte, la producción de $\mathrm{H}_{2}$ puede verse afectada por la descarboxilación de minerales de $\mathrm{CO}_{3}{ }^{-2}$ (carbonatos), lo cual aumenta la concentración de $\mathrm{CO}_{2}$ y genera que la reacción se desplace hacia la izquierda de la ecuación 1, reduciéndose la producción de $\mathrm{CO}_{2} \mathrm{y} \mathrm{H}_{2}$ (Roa, 2012). La fig. 7 muestra los procesos, subprocesos, reacciones y productos de la acuatermólisis catalítica.

$$
\mathrm{CO}+\mathrm{H}_{2} \mathrm{O} \leftrightarrow \mathrm{CO}_{2}+\mathrm{H}_{2}
$$

a)

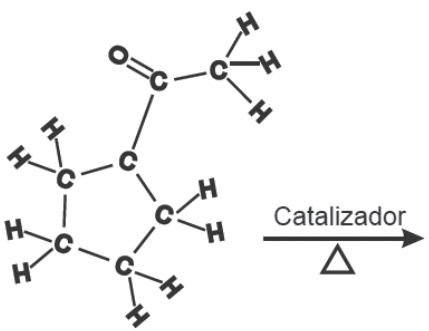

Ciclopentil-metil-centona

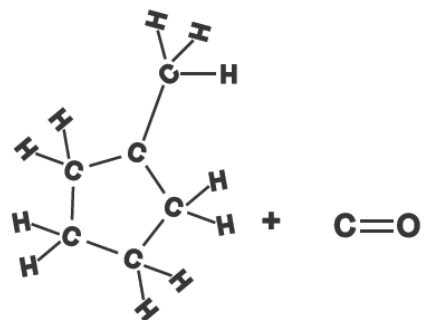

Metilciclopentano

Monóxido

de carbono

\section{b)}

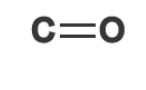

Monóxido de carbono
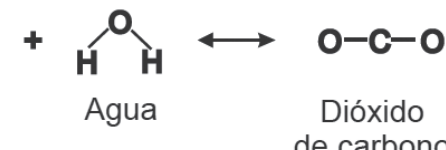
de carbono

Figura 6. Proceso de W.G.S. a) pirolisis y b) W.G.S. Fuente: Acevedo (2010).

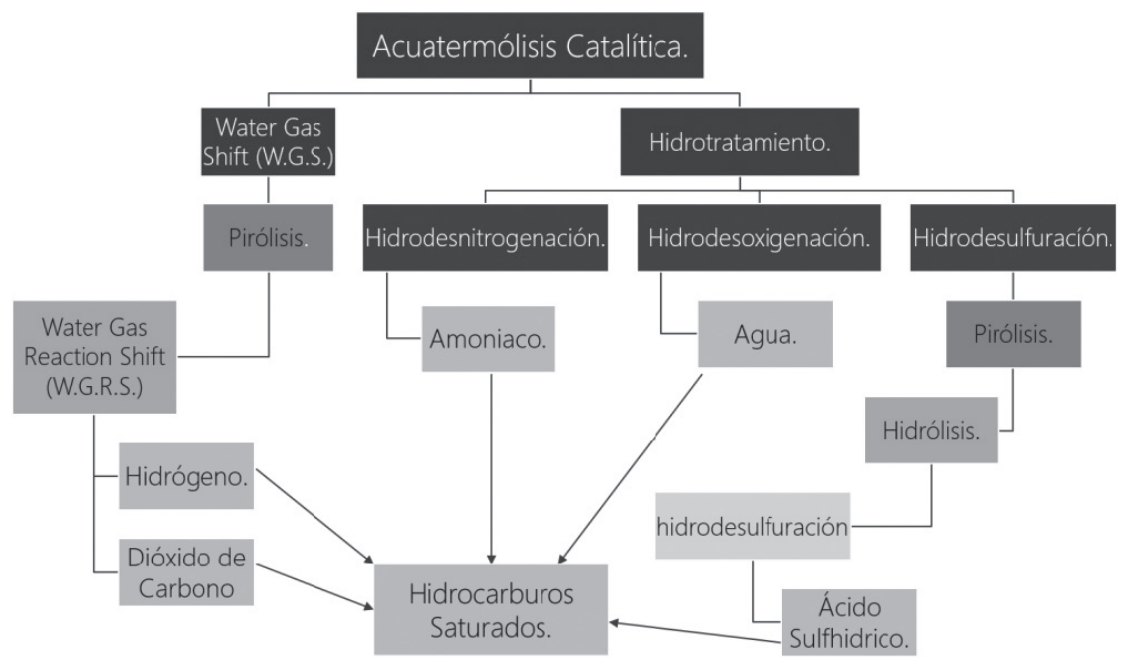

Figura 7. Síntesis de la acuatermólisis catalítica. 


\section{Aplicaciones del proceso}

Como ya se ha mencionado, las reacciones de acuatermólisis catalítica se dan principalmente entre las resina, los asfaltenos, y el vapor de agua. El estudio titulado "Cambio de las propiedades de resinas y asfaltenos después de la acuatermólisis catalítica" (Yufeng, Shuyuan, Fuchen \& Hang, 2006), busca confirmar dicha afirmación. Para ello se examina el efecto de cuatro catalizadores (dos solubles en agua y dos solubles en aceite) sobre las resinas o los asfaltenos, extraídas de petróleo proveniente de Liaohe (China), los cuales fueron separadas mediante cromatografía de adsorción líquido-sólido. Un análisis SARA realizado a este petróleo, muestra que su composición en peso de saturados, aromáticos, resinas y asfaltenos es de: $25.35 \%, 19.17 \%, 53.14 \%$ y $2.34 \%$ respectivamente. Para la experimentación se añadieron 5 gramos de asfaltenos o resinas a un reactor de acero inoxidable de $500 \mathrm{~mL}$ operado en lotes y equipado con agitador magnético. Se agregó $50 \mathrm{~mL}$ de ciclo-hexano como donador de hidrógeno. Para los catalizadores se tiene dos condiciones: la primera es utilizar un $0.1 \%$ en peso de catalizador soluble en aceite: nafteno de níquel NiN o nafteno de hierro FeN, y la segunda es agregar un $0.1 \%$ en peso de sulfato de níquel NiSO4 o sulfato ferroso FeSO4 como catalizador soluble en agua; posteriormente se añadió $500 \mathrm{~mL}$ de agua. Las condiciones de reacción fueron 48 horas a $280^{\circ} \mathrm{C}$; una vez terminado este tiempo de reacción se enfrió y los productos de petróleo y gas se recolectaron para posteriormente analizarlos.

El gas producido y la conversión de asfalteno o resina son calculadas mediante la ecuación 2 y 3 :

$\%$ Gas $=\frac{\text { Wasf.o.res.react }- \text { Wfas.acei.prod }}{\text { Wasf.o.res.react }} * 100 \%$

Donde:

-Wasf.o.res.react.: es la cantidad de asfalteno o resina de reactivo.

-Wfas.acei.prod.: es la cantidad de fase aceite en los productos.

$$
\% \text { Con.rea }=\frac{\text { Wasf.o.res.reac }}{\text { Wasf.o.res.react }} * 100 \%
$$

Donde:

-Wasf.o.reac.: es la cantidad de asfalteno o resina que reaccionó.

- Wasf.o.res.react.: es la cantidad de asfalteno o resina de reactivo.
Una vez realizado la acuatermólisis catalítica, se analizó el gas producido y el asfalteno o resina residual. La fig. 8 y 9 muestran la conversión de las muestras de asfaltenos (o resina) y la contribución de cada producto a dicha conversión. Los catalizadores solubles en aceite mostraron un mejor rendimiento que los solubles en agua. El orden del efecto en la conversión de mayor a menor es: $\mathrm{FeN}>\mathrm{NiN}>\mathrm{FeSO} 4>\mathrm{NiSO} 4$. Los asfaltenos se convirtieron en gas, saturados, aromáticos, resinas e insolubles en tolueno (coque), mientras que las resinas se convirtieron en gas, saturados, aromáticos, asfaltenos e insolubles en tolueno (coque).

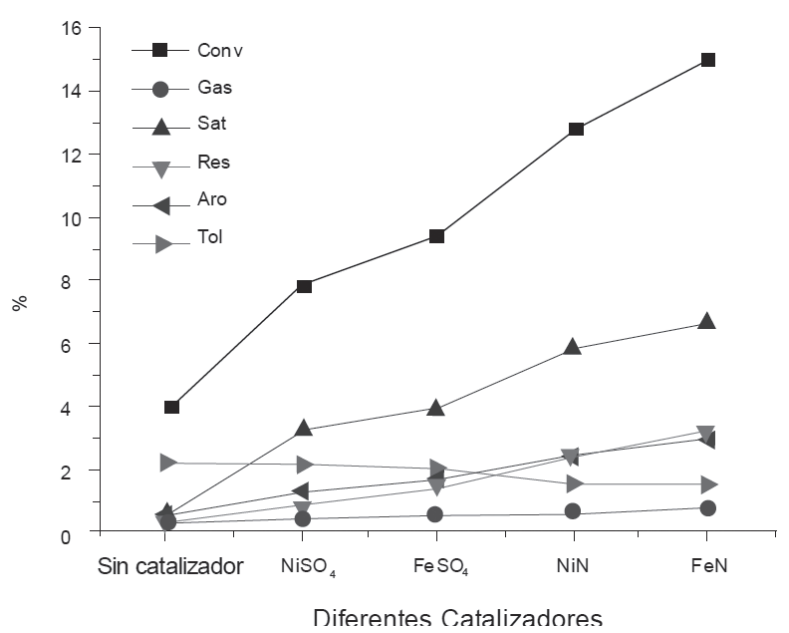

Figura 8. Conversión de asfaltenos y sus productos. Fuente: Yufeng (2006).

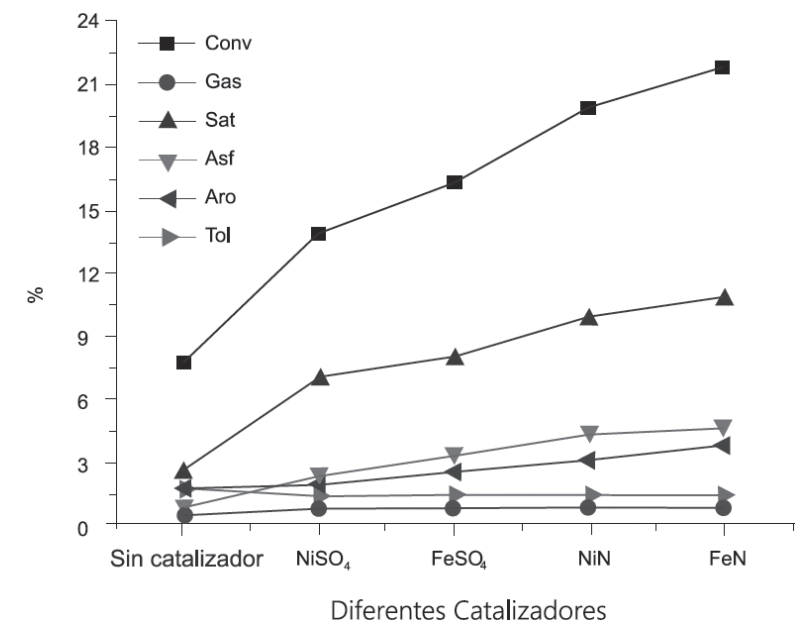

Figura. 9. Conversión de resinas y sus productos. Fuente: Yufeng (2006). 
La composición del gas obtenido después de realizada la acuatermólisis de asfaltenos y resinas con el uso o no de catalizadores es mostrada por las tablas 1 y 2 ; donde se evidencia un efecto positivo al implementar catalizadores ya que aumentan la proporción de hidrógeno y livianos, esto se interpreta en el mejoramiento de los procesos de hidrotratamiento y de W.G.S.

Tabla 1. Composición de gas de asfaltenos en acuatermólisis (\% v/v/). Fuente: Yufeng (2006).

\begin{tabular}{cccccccccc}
\hline Catalizador & $\mathbf{H}_{2}$ & $\mathbf{C O}$ & $\mathbf{C O}_{2}$ & $\mathbf{H}_{2} \mathbf{S}$ & $\mathbf{C H}_{4}$ & $\mathbf{C}_{2} \mathbf{H}_{6}$ & $\mathbf{C}_{2} \mathbf{H}_{4}$ & $\mathbf{C}_{3}+$ \\
\hline Sin-Catali. & 0 & 0 & 28.5 & 0.4 & 39.5 & 10.6 & 17.2 & 3.8 \\
NiSO4 & 1.3 & 0.5 & 21.7 & 0.3 & 43.0 & 13.6 & 14.8 & 4.8 \\
FeSO4 & 0.8 & 0.6 & 20.5 & 0.3 & 42.4 & 15.5 & 15.2 & 4.7 \\
\hline $\mathrm{NiN}$ & 1.5 & 1.2 & 22.8 & 0.2 & 40.4 & 18.5 & 12.4 & 3.0 \\
$\mathrm{FeN}$ & 1.1 & 1.3 & 24.1 & 0.2 & 37.2 & 19.6 & 13.7 & 2.8 \\
\hline
\end{tabular}

Tabla 2. Composición de gas de resinas de acuatermólisis (\%v/v). Fuente: Yufeng (2006).

\begin{tabular}{cccccccccc}
\hline Catalizador & $\mathbf{H}_{2}$ & $\mathbf{C O}$ & $\mathbf{C O}_{2}$ & $\mathbf{H}_{2} \mathbf{S}$ & $\mathbf{C H}_{4}$ & $\mathbf{C}_{2} \mathbf{H}_{6}$ & $\mathbf{C}_{2} \mathbf{H}_{4}$ & $\mathbf{C}_{3}+$ \\
\hline Sin-Catali. & 0 & 0 & 26.5 & 0.4 & 40.2 & 24.4 & 5.4 & 3.1 \\
NiSO4 & 0.9 & 1.5 & 21.4 & 0.2 & 42.7 & 22.8 & 7.7 & 2.8 \\
FeSO4 & 0.5 & 1.0 & 18.8 & 0.3 & 41.7 & 25.2 & 9.4 & 3.1 \\
NiN & 0.3 & 0.7 & 17.9 & 0.3 & 42.4 & 25.2 & 8.8 & 4.4 \\
FeN & 0.3 & 0.8 & 15.8 & 0.3 & 43.5 & 26.8 & 8.7 & 3.8 \\
\hline
\end{tabular}

El análisis elemental de las resinas y asfaltenos, la relación $\mathrm{H} / \mathrm{C}$, el contenido de oxígeno y azufre, antes y después de la acuatermólisis con el disminuye comoconsecuencia de la des-alquilación catalizador FeN se presenta en la tabla 3. En ella e hidrotratamiento. se evidencia que para las resinas y los asfaltenos

Tabla 3. Análisis elemental resinas y asfaltenos antes y después de la acuatermólisis con FeN. Fuente: Yufeng (2006).

\begin{tabular}{cccccccc}
\hline Muestra & $\mathbf{\% C}$ & $\mathbf{\% H}$ & $\mathbf{\% O}$ & $\mathbf{\% N}$ & $\mathbf{\% S}$ & H/C \\
\hline Resina antes acuater. & 85.25 & 10.45 & 2.41 & 1.04 & 0.85 & 1.47 \\
Resina desp. acuater. & 85.82 & 9.84 & 2.38 & 1.33 & 0.63 & 1.38 \\
Asfalteno antes acuater. & 86.31 & 9.05 & 2.33 & 0.96 & 1.35 & 1.26 \\
\hline Asfalteno desp. acuater. & 87.16 & 8.62 & 2.11 & 1.04 & 1.07 & 1.19 \\
\hline
\end{tabular}

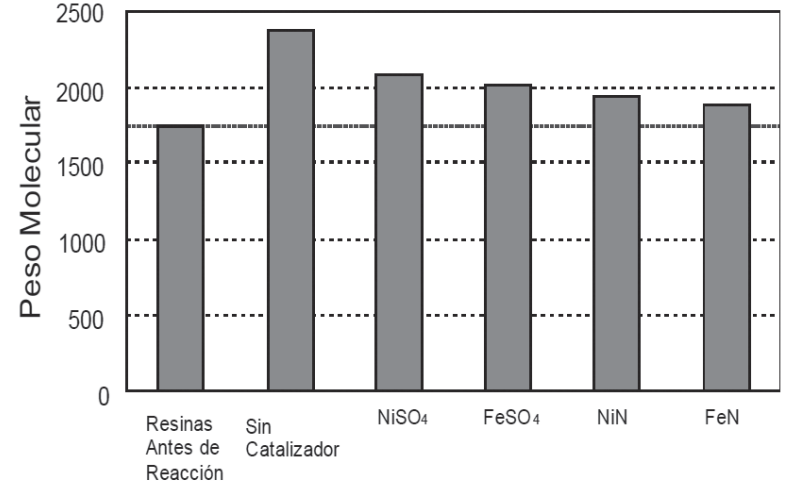

Diferentes Catalizadores

Figura 10. Peso molecular de las resinas antes y después de la acuatermólisis usando diferentes catalizadores. Fuente: Yufeng (2006).

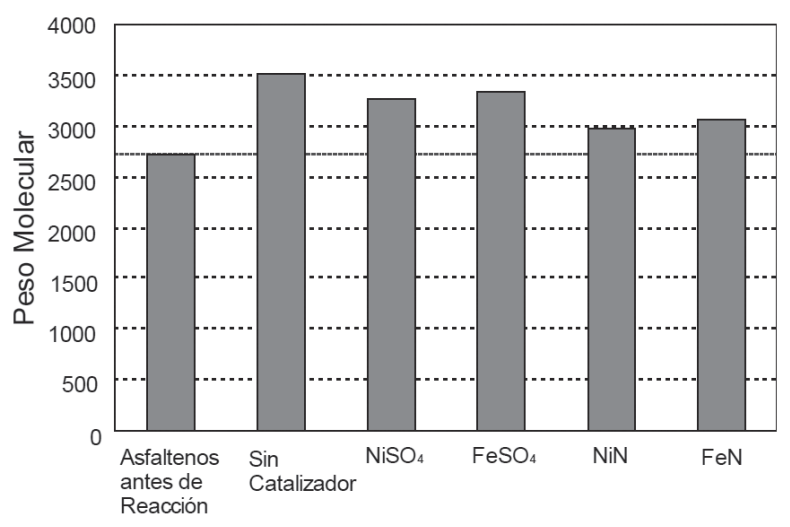

Diferentes Catalizadores

Figura 11. Peso molecular de los asfaltenos antes y después de la acuatermólisis usando diferentes catalizadores. Fuente: Yufeng (2006). 
En las fig. 10 y 11 se muestran el peso molecular medio de las resinas y asfaltenos, antes y después de la acuatermólisis con y sin catalizador. Contrario a lo que se podría pensar, la des-alquilación aumentó el peso molecular en vez de disminuirlo. Esto ocurre debido a que es inevitable la reacción de polimerización en el proceso. Sin embargo, como se observa, el uso de catalizador, mejora las reacciones de hidrogenación (hidrotratamiento) ya que disminuye el peso molecular comparado a si se usa solo vapor de agua sin catalizador.
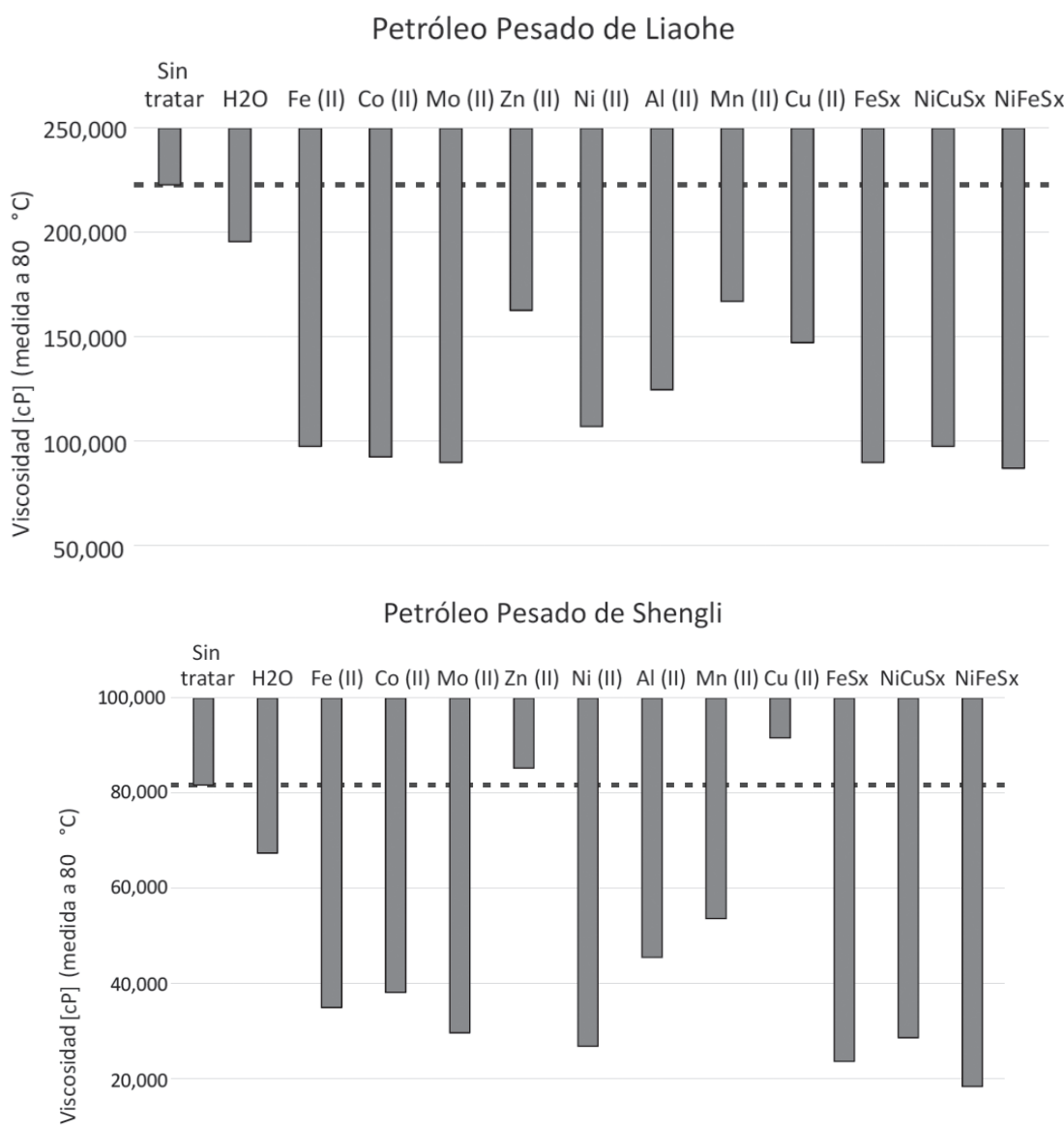

Figura 12. Efecto de las especies metáliscas sobre la viscosiadad del petróleo pesado de Liaohe y Shengli. Fuente: Jiang et al. (2005).

Tabla 4. Propiedades del petróleo pesado de Liaohe y Shengli, sin tratar y tratado. Fuente: Jiang et al. (2005).

\begin{tabular}{|c|c|c|c|c|c|c|}
\hline \multirow{2}{*}{ Ítem de análisis } & \multicolumn{3}{|c|}{ Petróleo pesado de Liaohe (\%reducción) } & \multicolumn{3}{|c|}{ Petróleo pesado de Shengli (\%reducción) } \\
\hline & Sin tratar & $\mathrm{Fe}$ (II) & $\mathrm{Fe}(\mathrm{II})+\mathrm{THN}$ & Sin tratar & $\mathrm{Fe}(\mathrm{II})$ & $\mathrm{Fe}(\mathrm{II})+\mathrm{THN}$ \\
\hline Viscosidad $[\mathrm{cP}]\left(\mathrm{a} 80^{\circ} \mathrm{C}\right)$ & 220000 & $96000(56.36 \%)$ & $28300(87.14 \%)$ & 84400 & $36200(57.11 \%)$ & $12000(85.78 \%)$ \\
\hline \multicolumn{7}{|l|}{ A. Elemental\%p } \\
\hline $\mathrm{C}$ & 86.80 & 86.60 & 86.20 & 85.30 & 85.50 & 85.70 \\
\hline $\mathrm{H}$ & 11.00 & 11.70 & 12.30 & 11.40 & 12.10 & 12.4 \\
\hline $\mathrm{S}$ & 0.96 & 0.63 & 0.32 & 1.80 & 1.10 & 0.77 \\
\hline $\mathrm{N}$ & 0.80 & 0.76 & 0.73 & 1.20 & 1.00 & 0.97 \\
\hline $\mathrm{H} / \mathrm{C}$ & 1.52 & 1.62 & 1.71 & 1.60 & 1.70 & 1.74 \\
\hline \multicolumn{7}{|l|}{ SARA, \%p } \\
\hline Saturados & 17.40 & 21.60 & 24.30 & 21.80 & 27.60 & 29.10 \\
\hline Aromáticos & 19.20 & 22.20 & 24.20 & 23.30 & 26.70 & 27.50 \\
\hline Resinas & 43.90 & 40.80 & 38.20 & 39.40 & 35.80 & 33.60 \\
\hline Asfaltenos & 19.50 & 15.40 & 13.30 & 15.50 & 10.90 & 9.80 \\
\hline
\end{tabular}


La acuatermólisis catalítica ha tenido grandes avances de investigación en china; allí se han efectuado pruebas piloto en campos de la cuenca de Liaohe. El estudio titulado "In-situ Upgrading de Petróleo Pesado por tratamiento de Acuatermólisis Bajo Condiciones de Inyección de Vapor" (Jiang, Liu X, Liu \& Zhong, 2005), busca determinar el efecto de catalizadores metálicos y donadores de hidrógeno comerciales y desarrollados por los autores, también el efecto del tiempo de reacción y la influencia de la temperatura en la acuatermólisis a condiciones de inyección de vapor en laboratorio y a escala piloto para dos crudos provenientes de: Liaohe Oilfield y Shengli Oilfield.

El primer experimento se llevó a cabo a una temperatura de $240{ }^{\circ} \mathrm{C}$ y un tiempo de reacción de 72 horas, con el objetivo determinar cuál de los catalizadores utilizados (11 especies) presenta una mayor reducción de la viscosidad (medida a $80{ }^{\circ} \mathrm{C}$ ). Los resultados son mostrados en la fig. 12.

Todas Las especies metálicas a excepción del Zn (II) y $\mathrm{Mn}$ (II) redujeron la viscosidad de ambos crudos. Para los siguientes experimentos se utilizó el Fe (II) en solución acuosa de concentración $0.02 \mathrm{M}$ y $0.04 \mathrm{M}$ en la mezcla de aceite, ya que presentó una reducción de más de la mitad de la viscosidad tiene un menor costo. Las tablas 4 y 5 , resumen las propiedades de las muestras antes y después del tratamiento con Fe (II) y el efecto sinérgico entre el $\mathrm{Fe}$ (II) y el donador de hidrógeno Tetralin (TNH).

Es evidente, en los resultados, que el catalizador de Fe (II) por sí solo genera cambios composicionales significativos en el crudo, pero existe una sinergia entre el catalizador de Fe (II) y el THN que hace más eficiente el proceso $y$, por ende, mejora las propiedades del crudo, donde se disminuye la viscosidad, aumenta la concentración de hidrocarburos saturados y aromáticos, y disminuye la concentración de resinas y asfaltenos. Por ejemplo, el petróleo pesado de Liaohe después de la aplicación de Fe (II) y THN en el proceso, se manifiesta que la concentración de hidrocarburos saturados y aromáticos pasó de $17,40 \%$ a $24,30 \%$ y $19,20 \%$ a $24,20 \%$, respectivamente; lo cual indica que la cantidad de hidrocarburos saturados aumentó en un $40 \%$ y los hidrocarburos aromáticos en un $26 \%$; por otra parte, la concentración de resinas y asfaltenos disminuyeron del $43.9 \%$ al $38.2 \%$ y del $19 \%$ al $13.3 \%$ respectivamente; es decir, la cantidad de resinas disminuyó en un $13 \%$ y de asfaltenos en un 30\%; los componentes entre el $\mathrm{C} 7 \mathrm{y}$ el C14 inicialmente eran del $48.33 \%$ pasaron a $66.77 \%$, un aumento del $38 \%$. Este cambio composicional origina que la viscosidad del crudo de Liaohe disminuya en un $87 \%$.

La fig. 13, muestra que el tiempo óptimo de reacción es de 72 horas, ya que para tiempos superiores no habría un cambio considerable en la viscosidad. La fig. 14, presenta el efecto de la temperatura de reacción sobre el proceso de acuatermólisis con o sin catalizador y/o donador de hidrógeno en el petróleo pesado de Liaohe. En un proceso de inyección de vapor, la temperatura oscila entre 160 y $280^{\circ} \mathrm{C}$ (Ahmed, 2012); y como era de esperarse la temperatura que mostró mayor reducción sobre la viscosidad del petróleo es de $260{ }^{\circ} \mathrm{C}$. Esto se debe a que a mayor temperatura mejores condiciones habrá para las reacciones de pirolisis.

Tabla 5. Efecto del Fe (II) y Fe (II) + THN en el petróleo pesado de Liaohe a $250^{\circ} \mathrm{C}$. Fuente: Jiang et al. (2005).

\begin{tabular}{|c|c|c|c|}
\hline \multirow{2}{*}{ Componentes } & \multicolumn{3}{|c|}{ Contenido, $\%$ p/p } \\
\hline & Sin tratar & $\mathrm{Fe}$ (II) & $\mathrm{Fe}$ (II) + THN \\
\hline $\mathrm{C} 7$ & 2.83 & 3.63 & 4.64 \\
\hline $\mathrm{C} 8$ & 4.58 & 4.94 & 5.63 \\
\hline C9 & 4.61 & 5.78 & 6.65 \\
\hline $\mathrm{C} 10$ & 6.08 & 7.90 & 7.98 \\
\hline $\mathrm{C} 11$ & 6.22 & 8.21 & 9.52 \\
\hline $\mathrm{C} 12$ & 6.85 & 9.05 & 9.89 \\
\hline $\mathrm{C} 13$ & 8.11 & 11.13 & 12.66 \\
\hline $\mathrm{C} 14$ & 9.05 & 10.35 & 9.80 \\
\hline $\mathrm{C} 15$ & 7.2 & 6.84 & 7.05 \\
\hline $\mathrm{C} 16$ & 5.82 & 5.61 & 5.73 \\
\hline $\mathrm{C} 17$ & 3.67 & 3.60 & 3.14 \\
\hline $\mathrm{C} 18$ & 6.37 & 3.75 & 3.11 \\
\hline C19 & 3.71 & 2.57 & 2.20 \\
\hline $\mathrm{C} 20$ & 5.03 & 3.42 & 2.52 \\
\hline $\mathrm{C} 21$ & 4.30 & 2.93 & 2.32 \\
\hline $\mathrm{C} 22$ & 4.21 & 2.35 & 1.47 \\
\hline $\mathrm{C} 23$ & 2.59 & 1.52 & 1.23 \\
\hline $\mathrm{C} 24$ & 2.52 & 2.29 & 1.36 \\
\hline $\mathrm{C} 25$ & 2.20 & 2.22 & 1.56 \\
\hline $\mathrm{C} 26$ & 2.06 & 1.35 & 1.21 \\
\hline $\mathrm{C} 27$ & 1.29 & 0.56 & 0.33 \\
\hline $\mathrm{C} 28$ & 0.70 & - & - \\
\hline
\end{tabular}

Una vez realizado el análisis de la acuatermólisis con catalizador y donador de hidrógeno se procede a realizar la aplicación en campo. En la tabla 6, se exponen los resultados de la aplicación en 20 pozos de Liaohe Oilfield los cuales fueron estimulados con vapor usando catalizador y donador de hidrógeno. 


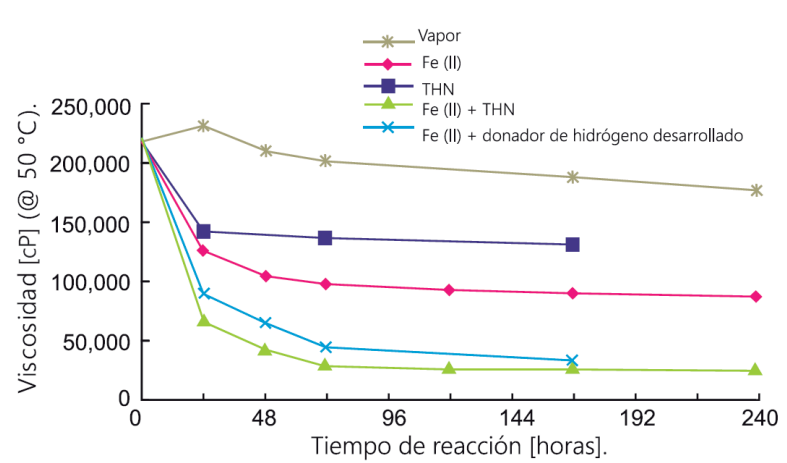

Figura 13. Efecto del tiempo de reacción en el petróleo pesado de Liaohe. Fuente: Jiang et al. (2005).

El resultado de la aplicación del tratamiento de acuatermólisis catalítica desde la evaluación técnica y tecnológica fue bueno, ya que incrementó en 68344 barriles la producción, un promedio de 3417 barriles por pozo; además se evidencia que el estilo de inyección "en la corriente" (intercurrent) tiene mayor impacto en la producción que el prelavado (preflush), ya que el el estilo "en la corriente" incremento en promedio la producción en $123 \%$ mientras que para el prelavado este incremento fue del $33 \%$. La tabla 7 , muestra un análisis elemental y un análisis SARA realizado en el pozo número 9; los

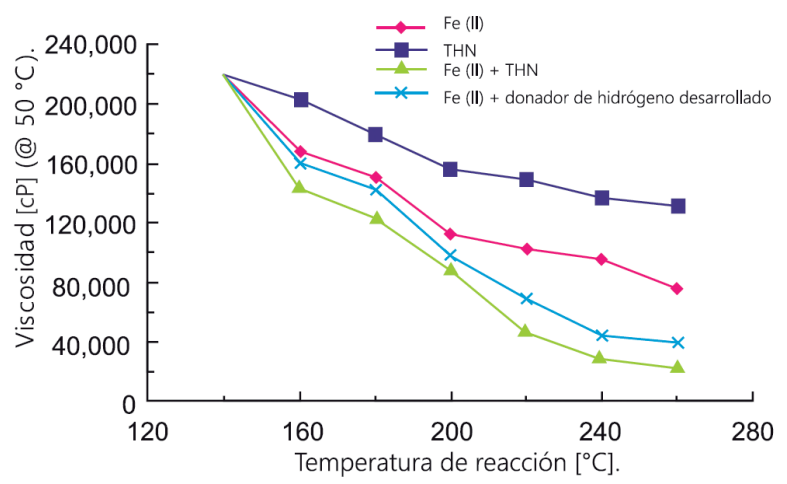

Figura 14. Efecto de la temperatura de reacción en el petróleo pesado de Liaohe. Fuente: Jiang et al. (2005).

resultados revelan que la cantidad de hidrocarburos saturados y aromáticos aumentaron en un 25\% y $23 \%$, respectivamente; mientras que las resinas y asfaltenos disminuyeron en un $8 \%$ y $28 \%$, respectivamente; la relación hidrógeno-carbono paso de 1.41 a 1.73 y la viscosidad se redujo en un $80 \%$. Estos valores fueron muy similares a los obtenidos en laboratorio a pesar de que en laboratorio se tiene mayor control de las variables como presión, temperatura, calidad del agua, volumen de agua por volumen de crudo, etc.

Tabla 6. Resultados evaluación de acuatermólisis catalítica en Liaohe Oilfield. Fuente: Jiang et al. (2005).

\begin{tabular}{|c|c|c|c|c|c|c|c|c|c|c|}
\hline \multirow{2}{*}{$\begin{array}{l}\text { No. } \\
\text { Pozo }\end{array}$} & \multirow{2}{*}{$\begin{array}{c}\text { Viscosidad a } 50 \\
{ }^{\circ} \mathrm{C},[\mathrm{cP}]\end{array}$} & \multirow{2}{*}{$\begin{array}{l}\text { Ciclo de } \\
\text { inyección }\end{array}$} & \multirow{2}{*}{$\begin{array}{l}\text { Estilo de } \\
\text { producción }\end{array}$} & \multirow{2}{*}{$\begin{array}{c}\text { Producción } \\
\text { de petróleo, } \\
\text { [Barril] }\end{array}$} & \multirow{2}{*}{$\begin{array}{l}\text { Producción } \\
\text { incremental } \\
\text { de petróleo, } \\
\text { [Barril] }\end{array}$} & \multirow{2}{*}{$\%$ incremental } & \multicolumn{2}{|c|}{ Relación SOR } & \multicolumn{2}{|c|}{$\begin{array}{c}\text { Relación de } \\
\text { agua }\end{array}$} \\
\hline & & & & & & & $\begin{array}{l}\text { Ciclo } \\
\text { antes }\end{array}$ & $\begin{array}{l}\text { Ciclo } \\
\text { desp. }\end{array}$ & $\begin{array}{l}\text { Ciclo } \\
\text { antes }\end{array}$ & $\begin{array}{l}\text { Ciclo } \\
\text { desp. }\end{array}$ \\
\hline 1 & 81070 & 4 & Prelavado & 6104 & 378 & 7 & 1.27 & 2.07 & 2.26 & 1.06 \\
\hline 2 & 112000 & 5 & Prelavado & 10784 & 1499 & 16 & 1.08 & 1.05 & 1.17 & 1.02 \\
\hline 3 & 10860 & 3 & Prelavado & 3600 & 1371 & 62 & 3.29 & 3.09 & 6.94 & 1.17 \\
\hline 4 & 98000 & 4 & En la corriente & 5984 & 3584 & 149 & 5.24 & 1.68 & 10.64 & 2.38 \\
\hline 5 & 10470 & 4 & En la corriente & 9121 & 1405 & 18 & 3.18 & 1.88 & 3.24 & 1.14 \\
\hline 6 & 10000 & 3 & En la corriente & 12657 & 5981 & 90 & 1.47 & 0.90 & 3.80 & 1.37 \\
\hline 7 & 230000 & 5 & Prelavado & 9273 & 1715 & 23 & 2.50 & 1.22 & 6.06 & 0.52 \\
\hline 8 & 330000 & 3 & Prelavado & 6774 & 2532 & 60 & 1.95 & 1.16 & 1.43 & 0.89 \\
\hline 9 & 660000 & 3 & En la corriente & 5146 & 4428 & 617 & 12.20 & 2.42 & 7.04 & 3.66 \\
\hline 10 & 359000 & 3 & En la corriente & 12856 & 4781 & 59 & 1.96 & 1.13 & 7.41 & 3.28 \\
\hline 11 & 224820 & 3 & En la corriente & 14897 & 5801 & 64 & 2.37 & 0.63 & 4.41 & 1.80 \\
\hline 12 & 399450 & 5 & En la corriente & 9637 & 4041 & 72 & 2.16 & 1.34 & 2.19 & 1.29 \\
\hline 13 & 300000 & 4 & En la corriente & 13494 & 5677 & 73 & 2.13 & 0.98 & 4.22 & 1.75 \\
\hline 14 & 320000 & 3 & En la corriente & 12058 & 4138 & 52 & 2.46 & 1.10 & 5.75 & 1.73 \\
\hline 15 & 78400 & 5 & En la corriente & 4264 & 2819 & 195 & 7.25 & 3.25 & 1.83 & 2.54 \\
\hline 16 & 4900 & 6 & En la corriente & 8330 & 5019 & 152 & 2.67 & 1.81 & 0.85 & 1.18 \\
\hline 17 & 5290 & 6 & En la corriente & 5591 & 3023 & 118 & 2.87 & 2.52 & 1.17 & 1.20 \\
\hline 18 & 4070 & 7 & En la corriente & 12369 & 5396 & 77 & 4.42 & 0.92 & 1.04 & 0.50 \\
\hline 19 & 3400 & 5 & En la corriente & 7132 & 2986 & 72 & 3.14 & 1.75 & 1.01 & 1.87 \\
\hline 20 & 4300 & 7 & En la corriente & 7543 & 1770 & 31 & 5.81 & 3.36 & 1.31 & 1.98 \\
\hline
\end{tabular}


Tabla 7. Análisis de una muestra de crudo de Liaohe del pozo No 9. Fuente: Jiang et al. (2005).

\begin{tabular}{|ccc|}
\hline Ítem analizado & Sin tratar & Tratado* \\
\hline Viscosidad $[\mathrm{cP}]\left(50{ }^{\circ} \mathrm{C}\right)$ & 660000 & 133000 \\
\hline Elemento de análisis, \%p & & \\
$\mathrm{C}$ & 86.8 & 85.8 \\
$\mathrm{H}$ & 10.2 & 12.4 \\
$\mathrm{~S}$ & 0.7 & 0.1 \\
$\mathrm{~N}$ & 0.8 & 0.3 \\
\hline Análisis SARA, \% p & & \\
\hline Saturados & 17.4 & 21.8 \\
Aromáticos & 19.2 & 23.7 \\
Resinas & 43.9 & 40.4 \\
Asfaltenos & 19.5 & 14.1 \\
\hline
\end{tabular}

*muestra cuando el pozo ha producido por 14 días.

\section{Conclusiones}

La industria petrolera mundial se mueve en la búsqueda de técnicas, tecnologías y en el estudio de procesos como la acuatermólisis catalítica que mejoran las propiedades del petróleo pesado y extrapesado in-situ. Este re direccionamiento se da por el agotamiento de las reservas de petróleo liviano y la gran cantidad de petróleo pesado y extrapesado.

Los procesos de W.G.S. e hidrotratamiento, abarcan las reacciones químicas de: pirolisis, hidrólisis, W.G.S.), hidrodesulfuración (HDS), hidrodesnitrogenación (HDN) e hidrodesoxigenación (HDO), las cuales generan una reducción del tamaño de las moléculas, como consecuencia se aumenta la concentración de saturados, se disminuye la concentración de resinas y asfaltenos, y se mejoran las propiedades físicas: viscosidad y densidad del crudo.

La reacción más importante de la acuatermólisis catalítica es W.G.S., en la cual la reacción de $\mathrm{CO}$ y $\mathrm{H}_{2} \mathrm{O}$ produce $\mathrm{CO}_{2} \mathrm{y} \mathrm{H}_{2}$. El $\mathrm{CO}_{2}$ es un gas que si se encuentra a condiciones adecuadas de presión y temperatura puede solubilizarse en el petróleo, esto disminuye la viscosidad y, por tanto, hace más fácil su extracción. Por otra parte, el $\mathrm{H}_{2}$ reacciona con los radicales producidos en la pirolisis, incrementando la relación $\mathrm{H} / \mathrm{C}$ y mejorando la calidad del crudo.

Usar solo catalizador, mejora las propiedades del petróleo; por ejemplo, la viscosidad. También cambia la composición y la concentración de heteroátomos. A pesar de ello, emplear catalizador y donador de hidrógeno a la vez, presentan un efecto sinérgico notablemente superior a la implementación de catalizador, donador de hidrógeno o vapor de agua en la acuatermólisis juntos o por separado.
La disminución de la concentración de $\mathrm{H}_{2} \mathrm{~S}$ en el gas proveniente del proceso de acuatermolósis en las resinas y asfaltenos cuando se usa o no se usa catalizador, es consecuencia del aumento de la concentración de $\mathrm{H}_{2}, \mathrm{CO}$, $\mathrm{CH}_{4}, \mathrm{C}_{2} \mathrm{H}_{6}, \mathrm{C}_{2} \mathrm{H}_{4}$ y otros hidrocarburos livianos. El hecho que la concentración de $\mathrm{H}_{2} \mathrm{~S}$ disminuya, no significa que el volumen producido sea menor; de hecho, la producción de gas se duplica con el uso de catalizador, por ende, a pesar de que la concentración de $\mathrm{H}_{2} \mathrm{~S}$ disminuya, la cantidad $\mathrm{H}_{2} \mathrm{~S}$ aumenta con el uso del catalizador lo que se interpreta como mejora en el hidrotratamiento.

El aumento de la concentración de $\mathrm{H}_{2}$ y $\mathrm{CO}$ se debe a que el uso del catalizador favorece las condiciones para la reacción de W.G.S.; mientras que la razón por la cual se incrementa de la concentración de $\mathrm{CH}_{4}, \mathrm{C}_{2} \mathrm{H}_{6}, \mathrm{C}_{2} \mathrm{H}_{4}$ en el gas, y saturados y aromáticos en la fase oleosa, se fundamenta en el hecho que el catalizador promueve el proceso de hidrotratamiento y las reacciones de desalquilación.

La relación hidrógeno-carbono de las resinas y los asfaltenos disminuyó después de la aplicación de la acuatermólisis catalítica, debido a que con la desalquilación se eliminan los componentes de mayor saturación presentes en las moléculas.

A pesar de que la reacción de desalquilación en el proceso debería disminuir el peso molecular, se muestra un fenómeno adverso en las resinas y asfaltenos producto de estas reacciones; ya que es inevitable la polimerización en la acuatermólisis, en especial cuando no se usa catalizador; a pesar de ello, utilizar catalizadores mejora las reacciones de W.G.S. y de donación de hidrógeno (hidrotratamiento) de tal manera que mitiga la polimerización y así el incremento abrupto del peso molecular.

La acuatermólisis catalítica muestra que su aplicación a escala de campo produce efectos similares en las propiedades del crudo a las obtenidas en laboratorio; por ejemplo la reducción de la viscosidad aproximadamente en laboratorio alcanzó el $90 \%$ y en campo el $80 \%$; esto a pesar que, en laboratorio se pueda mantener un mayor control de las condiciones y las variables que actúan sobre el proceso.

En la inyección cíclica de vapor, utilizar catalizadores mejoró las condiciones para que reaccione el vapor de agua, donador de hidrógeno y compuestos organosulfurados; esto es evidenciado por la producción de $\mathrm{H}_{2} \mathrm{~S}$, también fue exitosa, ya que incrementó la producción de petróleo hasta un $600 \%$ en el petróleo de Liaohe Oilfield. 


\section{Agradecimientos}

Al grupo de investigación de Recobro Mejorado (GRM-UIS) por ser parte fundamental de mi desarrollo científico e investigativo. Jorge Anderson Arboleda Lamus.

\section{Referencias}

1. Acevedo, L. (2010). Desarrollo de la Ingeniería Conceptual para la Aquathermolysis como Proceso para Mejoramiento de Crudo Pesado. Biblioteca Universidad Industrial de Santander (UIS) Proyecto de grado.

2.Alarcón,L.y Cavanzo,E.(2013). Estudio Experimental de un Proceso de Inyección Continua de Vapor en el Equipo de Desplazamiento Radial (E.D.R.) con Medios Porosos Homogéneos. Grupo de Investigación Recobro Mejorado. Biblioteca Universidad Industrial de Santander (UIS) Proyecto de grado.

3. Alboudwarej, H., Felix, J. \& Taylor, S. (2006), La Importancia del Petróleo Pesado. published by Schlumberger, 38-59.

4. Cañas, M. C. R., Ramirez, H. B., \& Navarro, S. F. M. (2014). Cinética de procesos de combustión in situ: Una discusión de los modelos cinéticos. Fuentes: El reventón energético, 12(1), 1.

5. Castillo, A. (2017). Estudio de la Producción de H2S para un Crudo Pesado Candidato a Inyección Continua de Vapor Mediante Caracterización Cuantitativa y Análisis Termodinámico de Compuestos Azufrados. Grupo de Investigación Recobro Mejorado. Biblioteca Universidad Industrial de Santander (UIS) Proyecto de grado.

6. Cavanzo, E. A., Navarro, S. F. M., Ramirez, H. B., Niz, E., \& Rodríguez, A. O. (2016). Combustión in situ húmeda: alternativa para el recobro mejorado en Colombia. Fuentes: El reventón energético, 14(1), 5-18.

7. Garzon, A. J. Y., Cañas, M. C. R., \& Navarro, S. F. M. (2012). Reacción y difusión: Dos factores claves en la técnica de combustión in situ. Fuentes: El reventón energético, 10(2), 5.

8. Gonzalez, C. (2014). Hidrodesoxigenación de Compuestos Aromáticos Oxigenados Sobre Catalizadores de Metal Precioso Soportado.
Biblioteca Universidad de Oviedo. Tesis Doctoral.

9. Jiang, S., Liu, X., Liu, Y. \& Zhong, L. (2005). In situ Upgrading Heavy Oil by Aquathermolytic Treatment Under Steam Injection Conditions. Society of Petroleum Engineers Inc.SPE 91973.

10. Kapadia, P., Kallos, M. \& Gates, I. (2014). A review of pyrolysis, aquathermolysis, and oxidation of Athabasca bitumen. Department of Chemical and petroleum engineering. . ELSEVIER, Fuel Processing Technology 131, 270-289.

11. Mercado, D. (2008). Modelo Analítico para Predecir el Comportamiento de la Inyección Continua de Vapor en Yacimientos Estratificados de Crudo Pesado. Grupo de Investigación RECOBRO MEJORADO. Biblioteca Universidad Industrial de Santander (UIS) Proyecto de grado.

12. Molina, A. (2015). Gráfico de la Semana de Campetrol La Era de Los Crudos Pesados en Colombia. Campetrol. Inteligencia Petrolera.. Recuperado de http://bit.ly/2uscyVr

13. Muraza, O. Y Galadima, A. (2015). Aquathermolysis of heavy oil: A review and perspective on catalyst development. . Department of Chemical and petroleum engineering. ELSEVIER, Fuel 157, 219-231.

14. Roa, L. (2012). Steam- Aqua Catalytic Upgrading of Bitumen at near In Situ Production Conditions. THESIS University of Calgary, Canada.

15. Rojas, J. F. F., Cañas, M. C. R., \& Navarro, S. F. M. (2012). Combustión in situ: Un estudio de reactores para representarla. Fuentes: El reventón energético, 10(2), 3.

16. Valdevenito, F. García, R., Escalona, N., Gilllambias, F., Rasmussen, S. Y López, A. (2010), NiMo Synergism Via Hydrogen Spillover, in Pyridine Hydrodenitrogenation.. ELSEVIER, Catalysis Communications 11, 1154-1156.

17. Villamarin, A. M. C., Cañas, M. C. R., \& Navarro, S. F. M. (2014). Modelado de procesos de combustion in situ. Fuentes: El reventón energético, 12(1), 5.

18. Wauquier, J. (2004). El refino del petróleo. Instituto Francés del Petróleo. Instituto superior de la energía (ISE). 
19. Yufeng, Y., Shuyuan, L., Fuchen, D. \& Hang, Y. (2009). Change of asphaltene and resin properties after catalytic aquathermolysis. Pet. Sci.6:194-200.

\section{Tabla de conversión de unidades}

\begin{tabular}{cccc}
\hline Cantidad & Nombre & Símbolo & Valor en S.I.U. \\
\hline \multirow{2}{*}{ Longitud } & Pie & $\mathrm{ft}$ & $30.48 \mathrm{E}-2 \mathrm{~m}$ \\
& Pulgada & in & $2.54 \mathrm{E}-2 \mathrm{~m}$ \\
\multirow{2}{*}{ Presión } & $\begin{array}{c}\text { Libra por pulgada } \\
\text { cuadrada }\end{array}$ & $\mathrm{psi}$ & $6894.76 \mathrm{~kg} / \mathrm{m}^{*} \mathrm{~s}^{2}$ \\
& Acre & $\mathrm{ac}$ & $4046.86 \mathrm{~m}^{2}$ \\
\multirow{2}{*}{ Área } & Pie cuadrado & $\mathrm{ft}^{2}$ & $9.290 \mathrm{E}-2 \mathrm{~m}^{2}$ \\
& Barril & $\mathrm{Bbl}$ & $119.24 \mathrm{~m}^{3}$ \\
Volumen & Galón & $\mathrm{gal}$ & $3.78 \mathrm{E}-3 \mathrm{~m}^{3}$ \\
& Libra & $\mathrm{Lb}$ & $453.59 \mathrm{E}-3 \mathrm{Kg}$ \\
\hline \multirow{2}{*}{ Masa } & Fahrenheit & ${ }^{\circ} \mathrm{F}$ & $5^{*}\left({ }^{\circ} \mathrm{F}-32\right) / 9{ }^{\circ} \mathrm{C}$ \\
\hline
\end{tabular}

Recepción: 15 de enero de 2018

Aceptación: 30 de marzo de 2018 